\title{
Research on the model of creative flat folding table
}

\author{
Yanbo Wang
}

North China Electric Power University, Huadian-Lu 689, 071000 Baoding,China

1046221363@qq.com

Keywords:multi objective programming, pressure bar stability, dynamic simulation, curve equation.

\begin{abstract}
In the subject one, we firstly number the legs of the table. According to the Pythagorean theorem, we can calculate the length of the table legs. Then describe the dynamic folding process of table by the change condition of angle between table leg and the ground, also we can understand the change of this angle during the process of folding; then we will calculate the distance between steel bar and the top of the table leg under the condition of normal status of and when it is under the usage respectively. Then we can solve the upper and lower boundary of the wooden slot of the table legs, as well as the length of slot; the establishment of rectangular coordinate system shall take the straight line where is the reinforce located as $\mathrm{X}$ axis, the vertical direction as the $\mathrm{Z}$ axis, the first wood reinforced position as the origin of coordinates. Representing the coordinates of each end of the batten with coordinate, then connecting each spot by using matlab, we can get the image of edge line of the table foot.
\end{abstract}

\section{Model assumptions}

1. Ignore the gap between the adjacent battens;

2.Wood is uniform, density is constant and the thickness is uniform.;

3.The thickness of the plank material used in the design is constant, which is $3 \mathrm{~cm}$

4.Ignore the thickness and width of the dynamic simulation

\section{Symbol Description}

L: $\quad$ Length of rectangular flat plate, unit: $\mathrm{cm}$

d: Width of table leg batten, unit: $\mathrm{cm}$

r: $\quad$ Radius of circular table, unit: $\mathrm{cm}$

$L_{i}$ : $\quad$ Length of Table leg No i , unit: $\mathrm{cm}, \mathrm{i}=1, \ldots, 10$

$C_{i}: \quad$ Upper boundary of Table leg No i unit: $\mathrm{cm}, \mathrm{i}=1, \ldots, 10$

$C_{i}: \quad$ lower boundary of Table leg No i unit: $\mathrm{cm}, \mathrm{i}=1, \ldots, 10$

$\theta_{i}$ : $\quad$ angle between batten and ground of Table leg No i, unit: $\mathrm{cm}, \mathrm{i}=1, \ldots, 20$,

$v_{i}$ : distance from steel to the top of the batten of Table leg No i under the normal condition. , unit: $\mathrm{cm}, \mathrm{i}=1, \ldots, 16$

\section{Establishment and Solution Model}

\section{1 solution of the length of table leg}

According to the symmetry of the folding table, it is only need to solve a $1 / 4$ root total number of wooden table leg length. The rectangular plate width is $50 \mathrm{~cm}$, every piece of wood is $2.5 \mathrm{~cm}$ of width, therefore the number of total wood root will be:

$$
(50 \div 2.5) \times 2=40 \text {, }
$$


Therefore, it is only need to calculate the length of $10 \operatorname{legs}^{[1]}$. The figure shows the plane rectangular coordinate system under the normal condition:

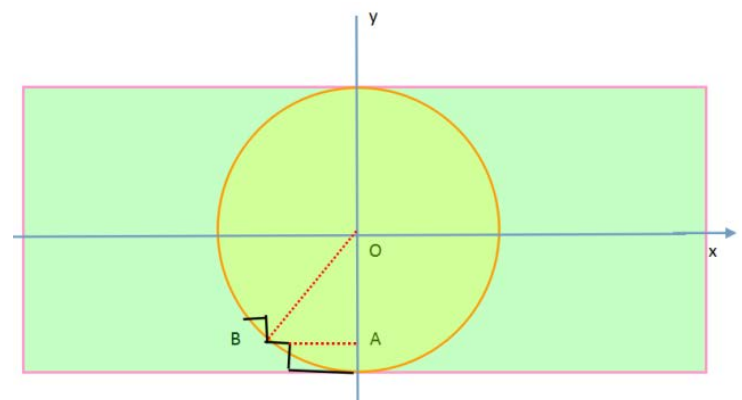

Fig. 1 The coordinate system is built when the folding table is in the flat plate state

Known round table radius is $\mathrm{r}=25 \mathrm{~cm}$, width of each batten is $\mathrm{d}=2.5 \mathrm{~cm}$, Rectangular plate length is $\mathrm{L}=120 \mathrm{~cm}$, number the 10 legs upward order as : $1,2,3,4,5,6,7,8,9,10$ 。

Triangle $0 A B$ is right angled triangle, $O A=r-d=22.5, O B=25$, according to the Pythagorean theorem can be obtained:

$$
A B=\sqrt{O B^{2}-O A^{2}}
$$

Length of second batten is :

$$
L_{2}=\frac{L}{2}-A B=\frac{L}{2}-\sqrt{O B^{2}-O A^{2}}
$$

Length of number $\mathrm{k}$ batten is $(\mathrm{k}=2, \ldots, 10)$ :

$$
L_{k}=\frac{L}{2}-\sqrt{r^{2}-(r-(k-1) d)^{2}}
$$

Thickness of rectangular plate is $3 \mathrm{~cm}$, consider the appearance of the folding table, we shall take the first stick length as $57 \mathrm{~cm}$

Therefore the length of 10 wooden batten will be:

\begin{tabular}{|l|l|l|l|l|l|l|l|l|l|l|}
\hline No & 1 & 2 & 3 & 4 & 5 & 6 & 7 & 8 & 9 & 10 \\
\hline $\begin{array}{l}\text { Len } \\
\text { gth/ } \\
\text { cm }\end{array}$ & $\begin{array}{l}57.0 \\
000\end{array}$ & $\begin{array}{l}49.10 \\
28\end{array}$ & $\begin{array}{l}45.000 \\
0\end{array}$ & $\begin{array}{l}42.146 \\
4\end{array}$ & $\begin{array}{l}40.000 \\
0\end{array}$ & $\begin{array}{l}38.349 \\
4\end{array}$ & $\begin{array}{l}37.087 \\
1\end{array}$ & $\begin{array}{l}36.151 \\
5\end{array}$ & 35.5051 & 35.1253 \\
\hline
\end{tabular}

\subsection{Dynamic change model}

Number the 10 legs on the left front from the front to the back as :1,2, .., 10, NR is the first batten, MS is the No $i(2 \leq i \leq 10)^{[2]}$. During the process of folding, the angle between NR line and the ground is NRF, the $\alpha$, The angle between MJ line and the ground is MJF $(2 \leq i \leq 10)^{[3]}$.

(1) when $\theta_{i}$ is acute angle, which is $M N<\frac{1}{2} R F$ we can get the figure by auxiliary line:

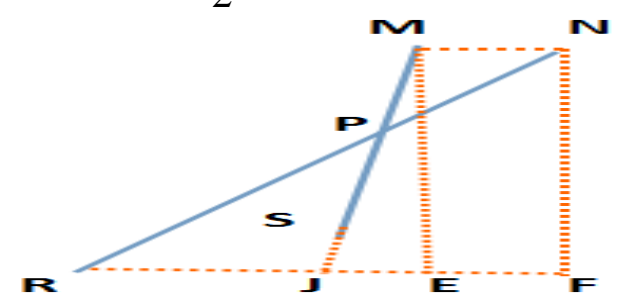

Fig.2 Dynamic change auxiliary line

$M E \perp R F, N F \perp R F, M N / / R F, N P=R P$ 。

According to the triangle similarity theorem, we can get: $M N=R J$, 
$M N=E F$,therefore, $J E=R F-R J-E F=R F-2 M N$,

Then $M N=L_{1}-L_{i}, \quad R F=L_{1} \cos \alpha, M E=N F=L_{1} \sin \alpha$, it comes to:

$$
\tan \theta_{i}=\frac{M E}{J E}=\frac{L_{1} \sin \alpha}{L_{1} \cos \alpha-2\left(L_{1}-L_{i}\right)}
$$

Namely, as for the batten that meet the conditions $L_{1}-L_{i}<\frac{1}{2} L_{1} \cos \alpha$ :

$$
\theta_{i}=\arctan \frac{L_{1} \sin \alpha}{L_{1} \cos \alpha-2\left(L_{1}-L_{i}\right)}
$$

(2) When $\theta_{i}$ is an obtuse angle, which is $M N>\frac{1}{2} R F$ we can get the figure by auxiliary line:

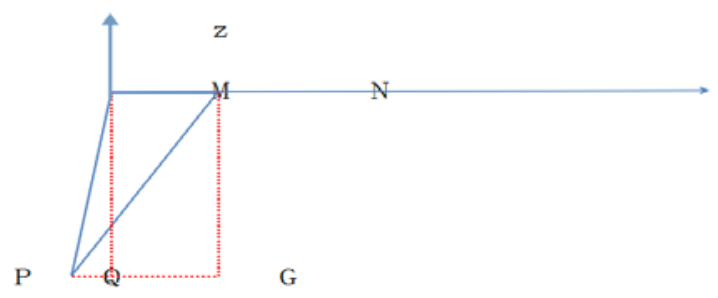

Fig.3 Dynamic change auxiliary line

$M E \perp R F, N F \perp R F, \quad M N / / R F, N P=R P$

According to the triangle similarity theorem, we can get: $M N=R J$,

$M N=E F$, therefore, $J E=R J+E F-R F=2 M N-R F$

Then $M N=L_{1}-L_{i}, \quad R F=L_{1} \cos \alpha, M E=N F=L_{1} \sin \alpha$, it comes to:

$$
\tan \theta_{i}=-\frac{M E}{J E}=\frac{L_{1} \sin \alpha}{L_{1} \cos \alpha-2\left(L_{1}-L_{i}\right)},
$$

Namely, as for the batten that meet the conditions $L_{1}-L_{i}>\frac{1}{2} L_{1} \cos \alpha$ :

$$
\theta_{i}=\pi+\arctan \frac{L_{1} \sin \alpha}{L_{1} \cos \alpha-2\left(L_{1}-L_{i}\right)} ;
$$

(3) when $\theta_{i}$ is a right angle, which is $M N=\frac{1}{2} R F, \theta_{i}=\frac{1}{2} \pi$,

for the batten that meet the conditions $L_{1}-L_{i}=\frac{1}{2} L_{1} \cos \alpha$, it is $\theta_{i}=\frac{1}{2} \pi$.

Also known: when the folding table is in the state of flat plate and the use of the state, the value of $\alpha$ are 0 and $\arcsin \frac{53}{57}$ respectively ${ }^{[4]}$.

Therefore, during the process of folding, the angle of wooden table legs and ground is: 


$$
\begin{aligned}
& \theta_{i}=\left\{\begin{array}{c}
\arctan \frac{L_{1} \sin \alpha}{L_{1} \cos \alpha-2\left(L_{1}-L_{i}\right)}, \quad L_{1}-L_{i}<\frac{1}{2} L_{1} \cos \alpha \\
\pi+\arctan \frac{L_{1} \sin \alpha}{L_{1} \cos \alpha-2\left(L_{1}-L_{i}\right)}, L_{1}-L_{i}>\frac{1}{2} L_{1} \cos \alpha \\
\frac{\pi}{2}, L_{1}-L_{i}=\frac{1}{2} L_{1} \cos \alpha
\end{array}\right. \\
& \text { while, } L_{1}=57, L_{\mathrm{m}}=\frac{L}{2}-\sqrt{r^{2}-(r-(m-1) d)^{2}} \quad(\mathrm{~m}=2, \ldots, 10), \quad 2 \leq i \leq 10,0 \leq \alpha \leq \arcsin \frac{53}{57} \text {. }
\end{aligned}
$$

Schematic diagram of dynamic change process:
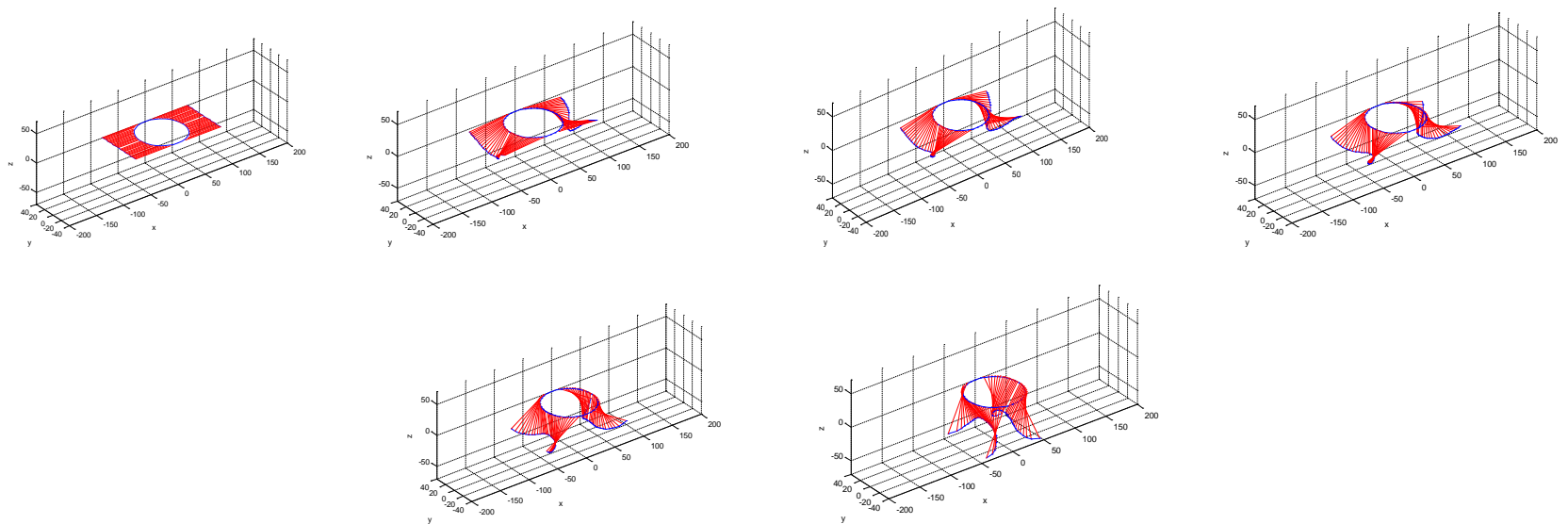

Fig.4 Sketch map of dynamic changing process of folding table

\section{Summary}

According to the relevant knowledge of analytic geometry, the mathematical model of the dynamic change coordinate of the folding table is determined, and the problem is solved well.

But, in the process of building the model, without considering the thickness of the reinforcement, but only as a straight line, will make the results have a certain error;

Stability analysis, only from the effect of the effect of force to consider the overall situation.

The steel bar can be abstracted as its central axis, which can reduce the error and make the model more close to the fact ${ }^{[5]}$.

\section{Reference}

[1].Huizu Shan. Mechanics of materials. Higher education press,.2010

[2].Weisheng Qiu. Analytic geometry. Peking University press

[3].Xijing Sun,Shoukui Si. Mathematical modeling algorithms and applications. National Defense Industry Press.

[4] Yu Xiaohong, Lin Xiuzhen, Zhang Fan, Wang Xudong. The design points of chair type furniture [J]. wood processing machinery. 2003 (06)

[5] Yang Zhimin, Zhang Yachi, Zhang Shuangbao. Application of ergonomics in chair design [J]. wood processing machinery. 2002 (04) 